\title{
Motion corrected fetal body MRI provides reliable 3D lung volumes in normal and abnormal fetuses
}

\author{
Joseph Davidson ${ }^{1}$, Alena Uus ${ }^{2}$, Alexia Egloff Collado², Milou Van Poppel ${ }^{2}$, Jacqueline \\ Matthew $^{2}$, Johannes Steinweg ${ }^{1}$, Maria Deprez ${ }^{2}$, Michael Aertsen ${ }^{3}$, Jan Deprest ${ }^{4}$, and Mary \\ Rutherford $^{2}$ \\ ${ }^{1}$ Evelina London Children's Hospital \\ ${ }^{2}$ King's College London School of Biomedical Engineering and Imaging Sciences \\ ${ }^{3}$ Universitaire Ziekenhuizen Leuven \\ ${ }^{4}$ Center for Surgical Technologies
}

September 24, 2021

\begin{abstract}
Abstract Objective:Evaluate deformable slice-to-volume registration (DSVR) to calculate 3D-segmented total lung volume (TLV) in fetuses with congenital diaphragmatic hernia, congenital lung lesions and healthy controls, with comparison to 2Dmanual segmentation. Design:Pilot study Setting:Regional fetal medicine referral centre Sample:Fetal MRIs performed for clinical indications (abnormal cases) or as research participants (healthy controls) Methods:Sixteen MRI datasets of fetuses (22-32 weeks GA). Diagnosis: $\operatorname{CDH}(n=5), \operatorname{CPAM}(n=2), \operatorname{CDH}$ with $\operatorname{BPS}(\mathrm{n}=1)$ and healthy control(n=8). DSVR was used for reconstruction of $3 \mathrm{D}$ isotropic $(0.85 \mathrm{~mm})$ volumes of fetal body followed by semi-automated lung segmentation. The resulting $3 \mathrm{D}$ TLV were compared to the traditional 2D-based volumetry, and a normogram of DSVR-derived fetal lung volumes from 100 cases was produced. Main Outcome Measures:Concordance with 2D-volumetry assessed with Bland-Altman analysis, results of segmentations presented visually. Observed/Expected values were calculated for abnormal cases based upon the normogram. Results:DSVR-derived TLV values have high correlation with the 2D-based measurements but with a consistently lower volume; bias $-1.44 \mathrm{~cm} 3$ [95\% limits: -2.6 to -0.3$]$ with improved resolution able to exclude hilar structures even in severe motion corruption or in cases of lung hypoplasia. Conclusions:Application of DSVR for fetal MRI provides a solution for analysis of motion corrupted scans and does not suffer from the interpolation error inherent in 2D-segmentation as per current clinical practice. It increases information content of acquired data in terms of visualising organs in 3D space and quantification of volumes, which we believe will have important value for counselling and surgical planning. Keywords:Fetal MRI; congenital diaphragmatic hernia; CPAM; lung volume
\end{abstract}

\section{Motion corrected fetal body MRI provides reliable 3D lung volumes in normal and abnormal fetuses}

Joseph Davidson ${ }^{1,2,3} \S$, Alena Uus ${ }^{4} \S$, Alexia Egloff ${ }^{5}$, Milou van Poppel ${ }^{4,6}$, Jacqueline Matthew ${ }^{4}$, Johannes Steinweg ${ }^{4,6}$, Maria Deprez ${ }^{4}$, Michael Aertsen ${ }^{7}$, Jan Deprest ${ }^{2,8,9}$, Mary Rutherford ${ }^{5}$

1. Department of Paediatric Surgery, Evelina Children's Hospital, London, United Kingdom

2. Elizabeth Garrett Anderson Institute of Women's Health, University College London, London, United Kingdom

3. GOS-UCL Institute of Child Health, London, United Kingdom

4. Biomedical Engineering Department, School of Biomedical Engineering and Imaging Sciences, King's College London, London, United Kingdom 
5. Centre for the Developing Brain, School of Biomedical Engineering and Imaging Sciences, King's College London, London, United Kingdom

6. Department of Congenital Heart Disease, Evelina Children's Hospital, London, United Kingdom

7. Department of Imaging and Pathology, Clinical Department of Radiology, University Hospitals KU Leuven, Leuven, Belgium.

8. Clinical Department of Obstetrics and Gynaecology, University Hospitals Leuven, Leuven, Belgium

9. Academic Department of Development and Regeneration, Cluster Woman and Child, KU Leuven, Leuven, Belgium

$\S$ contributed equally to this manuscript

\section{Abstract}

Objective: To evaluate deformable slice-to-volume registration (DSVR) to calculate 3D-segmented total lung volume (TLV) in fetuses with congenital diaphragmatic hernia, congenital lung lesions and healthy controls, with comparison to 2D-manual segmentation. To establish normogram of TLV calculated by DSVR in health control fetuses.

Design: Pilot study

Setting: Single regional fetal medicine referral centre

Sample:Fetal MRIs performed for clinical indications (abnormal cases) or as research participants (healthy controls)

Methods: Sixteen MRI datasets of fetuses (22-32 weeks GA). Diagnosis: $\mathrm{CDH}(\mathrm{n}=6$, one with coexisting BPS $), \operatorname{CPAM}(\mathrm{n}=2)$, and healthy controls $(\mathrm{n}=8)$. DSVR was used for reconstruction of 3D isotropic $(0.85$ $\mathrm{mm}$ ) volumes of the fetal body followed by semi-automated lung segmentation. The resulting 3D TLV were compared to the traditional 2D-based volumetry, and abnormal cases referenced to a normogram produced from 100 normal fetuses whose TLV was calculated by DSVR.

Main Outcome Measures: Concordance with 2D-volumetry assessed with Bland-Altman analysis, results of segmentations presented visually. Observed/Expected values were calculated for abnormal cases based upon the normogram.

Results: DSVR-derived TLV values have high correlation with the 2D-based measurements but with a consistently lower volume; bias $-1.44 \mathrm{~cm}^{3}$ [95\% limits: -2.6 to -0.3] with improved resolution able to exclude hilar structures even in severe motion corruption or in cases of very low lung volumes. lung hypoplasia.

Conclusions: Application of DSVR for fetal MRI is beneficial for the analysis of motion corrupted scans and does not suffer from the interpolation error inherent in currently applied 2D-segmentation as per current clinical practice. It increases information content of acquired data in terms of visualising organs in 3D space and quantification of volumes, which we believe will improve counselling and surgical planning.

Keywords: Fetal MRI; congenital diaphragmatic hernia; congenital lung lesion; congenital pulmonary airway malformation; bronchopulmonary sequestration; lung volume

\section{Introduction}

The antenatal work-up of fetal thoracic anomalies increasingly includes fetal MRI in recent years. Fetal MRI aids in cases of diagnostic uncertainty by detailed delineation of soft tissues within the fetal chest, differentiating lung, bowel and liver more easily than ultrasound ${ }^{1}$, helping in the assessment of congenital diaphragmatic hernia (CDH) and congenital lung lesions (CLL, including congenital pulmonary airway malformation $[\mathrm{CPAM}]$ and bronchopulmonary sequestration [BPS]). The currently standardised prognostic markers for CDH and CPAM are the observed/expected Lung Head Ratio $\left(\mathrm{O} / \mathrm{E}\right.$ LHR, $\left.{ }^{2}\right)$ and the CPAM volume ratio $\left(\mathrm{CVR},{ }^{3}\right)$, both measured by ultrasound. Both of these metrics utilise single plane measurements 
of aspects of the fetal chest to extrapolate volumes. Although these metrics are valid surrogates for total lung volume $\left(\mathrm{TLV},{ }^{4}\right)$, generation of such values may have inherent intra- and inter-operator variability ${ }^{5}$. Discrepancies between 2D ultrasound and MRI-derived TLV measurements have been shown to be principally related to the failure to account for the contribution of the ipsilateral lung ${ }^{6}$. Furthermore, ultrasound-based imaging struggles with maternal habitus and fetal positioning, as well as extremes of liquor volume. 3D ultrasound lung volume measurements have been shown to be more difficult than by MRI, mainly because the most hypoplastic lung cannot be properly visualized ${ }^{7}$. High volume fetal medicine centres have suggested that MRI-calculated lung volumes may be a more accurate predictor of survival but that has not been formally proven ${ }^{8}$, however there is no standardised methodology for the use of MRI in these cases which limits the compilation of large datasets necessary for further study ${ }^{9}$.

Modern clinical fetal MRI protocols (primarily based on single shot turbo spin echo (ssTSE) sequence) allow fast acquisition of individual 2D slices that "freeze" fetal position in time. These slices have sufficiently high image and contrast resolution for diagnostic purposes in cases where fetal motion may previously have limited the information available. However, the misalignment between individual slices leads to corruption of volumetric information and loss of structural continuity within a slice stack. Therefore, output MRI stacks are termed "motion corrupted". In general, to achieve a sufficient coverage of fetal visceral organs, clinical examinations require 3-6 MRI stacks acquired under different orientations with respect to the fetal body. The degree of motion corruption (misalignment of slices) can vary between the stacks and normally depends on the gestational age, amount of amniotic fluid, as well as fetal lie and mobility. It directly influences the accuracy of the fetal lung volume assessment, which is based on 2D slice-wise segmentation of motioncorrupted stacks followed by 3D interpolation. Therefore, calculated TLV values might vary for different stacks (from the same acquisition / the same subject) depending on the amount of motion corruption and acquisition plane. This is considered one of the limiting factors for MRI-based TLV assessment.

The recently proposed deformable slice-to-volume registration (DSVR) method ${ }^{10}$ is used for reconstruction of high-resolution (e.g., $0.8 \times 0.8 \times 0.8 \mathrm{~mm}$ ) isotropic 3D images of fetal body from multiple low-resolution (e.g., $1.25 \times 1.25 \times 1.25 \mathrm{~mm}$ ) motion-corrupted stacks. In DSVR method, one of the low-resolution stacks is selected as an initial target space and it is then registered to each of the slices using nonlinear free form deformation registration. This is followed by super-resolution reconstruction (SR) of the 3D image from the registered slices. The full pipeline includes 3 interleaved SR and SVR steps. The resulting reconstructed images provide detailed 3D volumetric information and can be reoriented in any plane. This facilitates accurate 3D segmentations of fetal lungs and other organs allowing volumetric analysis.

In this work, we sought to explore how DSVR-derived lung volumes would compare to those calculated by conventional manual 2D segmentation in cases of fetal thoracic anomalies, with a comparison to normal control cases.

\section{Methods}

\section{Case Selection}

The investigated cohort for 2D vs. 3D TLV assessment included 8 cases with abnormal lungs and 8 control cases from the iFIND project [https://www.ifindproject.com] and clinical fetal cardiac MRI databases. The abnormal cases included 6 cases of CDH (one with concomitant BPS), and a further 2 cases of CLL (both CPAM). The eight control cases were healthy control pregnancies and spanned approximately the same gestational range as the abnormal cases (22-32 weeks gestational age). In addition, 100 normal cases imaged as healthy control participants for research purposes from 21-33 weeks gestational age were used for the general assessment of 3D DSVR-derived fetal lung volumetry growth chart. All datasets used in this research were collected and processed subject to the informed consent of the participants.

\section{Data}

Each fetus was scanned using T2-weighted ssTSE sequence, producing between 6 and 10 stacks in different planes, with varying degrees of motion corruption. In all cases, images were acquired on an Ingenia 
1.5 Tesla (Philips) system using T2w ssTSE: TR $=15000 \mathrm{~ms}$, TE $=80 \mathrm{~ms}$, voxel size $1.25 \times 1.25 \mathrm{~mm}$, slice thickness $2.5 \mathrm{~mm}$ and spacing $1.25 \mathrm{~mm}$. For the initial 16 study cases, 2D slice-wise segmentation of the fetal lungs was performed in ITK-SNAP (http://www.itksnap.org) in the axial stack with respect to the fetal trunk and a total fetal lung volume was calculated using the conventional interpolation approach. These datasets also underwent DSVR reconstruction to $0.85 \times 0.85 \times 0.85 \mathrm{~mm}$ resolution using SVRTK toolbox (https://github.com/SVRTK/SVRTK) and lung volumes were calculated after automated 3D segmentation followed by manual refinement in 3D Slicer (https://www.slicer.org). An example of one of the he original 2D axial stacks affected by minor to moderate motion and the corresponding 3D DSVR reconstruction are displayed side by side for descriptive purposes in Figure 1 and Supplementary Video 1. The processing of the additional 100 cases included reconstruction using the standard DSVR pipeline ${ }^{10}$ followed by semi-automated lung segmentation.

Lung volumes from the 16 cases derived from 3D DSVR and the conventional 2D-based approach underwent pairwise Bland-Altmann comparison to assess for bias in measurement. In cases of CLL, lesion volume was calculated and is provided here in $\mathrm{cm}^{3}$ and as a proportion of TLV. A normal curve (growth chart) for fetal lung volumes was generated for 21-32 weeks GA range from 100 DSVR reconstructions, and this curve was compared descriptively to the previously published MRI-generated fetal lung volume normograms ${ }^{11,12}$.

\section{Results}

The examples of 2D vs. 3D lung segmentations for 4 cases are depicted in Figure 2; motion corruption of the axial-plane stack can clearly be appreciated in the coronal plane, with the coronal section of the DSVR shown alongside each case. DSVR was able to produce clear images even from severely motion-corrupted stacks (Figure 2C) and the resultant images can be examined in any plane and at a higher resolution than that of the original input stack (Figure 1, Video 1). The resultant segmentation of fetal lungs demonstrates a smooth outline, with normal cases depicting sufficient detail even to appreciate the lingula (Figure 2A) or a systemic feeding vessel in cases of CLL (Figure 2B). Comparison of the volumes generated from 2D and 3D segmentation is made in Figure 3; results correlated strongly, however a consistently lower measurement was made with 3D segmentation (Bland-Altman: Bias $-1.44 \mathrm{~cm}^{3}$ [95\% confidence limits -2.63 to -0.24$]$ ). This was felt to be due to the enhanced ability to exclude structures of the vascular hilum in $3 \mathrm{D}$ reconstructions, as well as an inherent reduction in interpolation error.

Having defined the fidelity of DSVR-derived 3D TLV, we proceeded to produce a normal curve based upon 100 healthy cases undergoing fetal MRI as normal controls for research, presented in Figure 4. The corresponding fitted TLV normogram has a high correlation with the previously reported gestational TLV curves produced from conventional 2D segmentation data by Cannie et $\mathrm{al}^{11}$ and Meyers et $\mathrm{al}^{12}$. The generated regression line had a similar $\mathrm{R}^{2}(0.72)$ to those published and was subsequently used to generate the "Expected" value for the depiction of Observed:Expected total lung volume in the abnormal cases.

The manually segmented 2D lung volumes and corresponding DSVR lung volumes are depicted graphically in Figure 5 with reference to the DSVR-derived normal curve. Table 1 displays data calculated from anomaly cases. CPAM lesion volumes were calculated from DSVR reconstructions and are presented as a percentage of the remaining TLV; of note one case had CDH and a BPS of the lung on the same side as the hernia, this case had significantly smaller lesion volume related to lung volume.

\section{Discussion}

This study demonstrates that MRI of the fetal thorax can be processed by 3D DSVR reconstruction, and subsequent lung volumetry can be performed with similar volumes generated to the 2D-manual segmentation derived volumes currently used in clinical practice for O/E TLV assessment. Furthermore, 3D lung segmentations potentially provide more accurate TLV estimation since they minimise the errors on the conventional 2D-based approach affected by the combination of misalignment of slices and interpolation. The analysis of the quantitative comparison results as well as the general visual inspection of $2 \mathrm{D}$ vs. 3D segmentations suggest that using 3D DSVR-based TLV assessment may potentially resolve one of the current limitations to the validity of MRI derived volumes in predicting outcomes as reliably as LHR $^{13}$. This series demonstrates 
that DSVR method provides the means for accurate calculation of lung volumes for both normal and abnormal cases. This is especially useful for $\mathrm{CDH}$ cases for more accurate evaluation of the observed to expected lung volume ratios. The recent advances in image processing methods reduce variability in segmentation ${ }^{14}$, further improving the reliability of the volumetry results. We anticipate that MR based lung volumetry may soon be validated as a reliable and more accurate prognostic indicator than the current use of the LHR; since it would invariably more closely predict true TLV. Furthermore, the ability to outline lesion volumes in cases of CLL will likely produce a prognostic marker more faithful to true lesion size than the currently utilised CVR where cross sectional areas are utilised to estimate lesion volume relative to fetal size. The consistency of such a method will certainly deliver on the recent plea for consistency in prognostication of these $\operatorname{cases}^{9}$. It should be noted that although we have provided lesion volume proportionate to TLV, it would be possible to segment any aspect of the fetus and derive a corresponding volume ratio proportional to this as well as the relative position of the organs (e.g., the degree of liver herniation or position of the stomach in CDH cases)

Recently, it has also been demonstrated that DSVR reconstructed MRI images may provide additional diagnostic information for complex fetal anomalies ${ }^{1}$. The illustrative cases included within this dataset also show that DSVR will produce datasets that are more accessible to the clinician by resolving motion corruption with a resolution superior to that of the original input stacks. This will certainly aid in prenatal counselling and planning of the operative approach in neonatal thoracic surgery; the reliable depiction of systemic feeding vessels in BPS, combined with US Doppler assessment may reduce the reliance on early postnatal CT imaging ${ }^{15}$.

Regarding longitudinal assessment of CDH or CLL, it should be recognised that the repeated use of MRI is considerably more expensive, time consuming and lengthy compared to an expert-performed ultrasound. Therefore, we anticipate that there will continue to be a role for ultrasound in the monitoring of such conditions to delivery. Indeed, serial CVR assessment has recently been shown to be effective in predicting a need for perinatal intervention in cases of CLL ${ }^{16}$.

\section{Conclusions}

This pilot study demonstrates the potential of a 3D reconstruction platform such as DSVR in the analysis of the fetal lung, with an emphasis on accuracy and reliability in image derived volumetry. We anticipate that MR based lung volumetry may soon be validated as a reliable and more accurate prognostic indicator than the current use of the LHR.

Our current work focuses on deep learning methods in order to provide reliable volumetric assessment for a wide range of anomalies as well as optimisation of different MRI acquisition protocols. This will allow integration of both DSVR and volumetry pipelines in different clinical centres that employ fetal MRI on a regular basis.

\section{Acknowledgements}

The iFIND study, from which these cases are derived, was supported by a Wellcome Trust IEH Award (102431). Research carried out at King's College London is made possible by NIHR funding of the Biomedical Research Centre; the views expressed here are those of the authors and not necessarily those of the NHS, NIHR or DoH.

\section{Disclosure of Interest Statement}

None of the authors have any conflict of interest to declare.

\section{Author Contribution}

JD and MR designed the study, analysed and interpreted the data, drafted the manuscript. AU designed the study, acquired the data, drafted the manuscript. AE, MvP, JM, JS acquired data and provided critical review of the manuscript. MD, MA, JD interpreted the data and provided critical review of the manuscript. 
All authors approved the final version for publication and agree to be accountable for all aspects of the work presented here.

\section{Details of Ethics Approval}

This study received national ethics approvals through the NHS REC: [07/H0707/105] in 2007 and [14/LO/1806] in 2014. All participating pregnant women taking part consented for the use of their imaging data in research.

\section{Funding Statement}

This study received no specific internal or external funding

\section{References}

1. Davidson JR, Uus A, Matthew J, Egloff AM, Deprez M, Yardley I, et al. Fetal body MRI and its application to fetal and neonatal treatment: an illustrative review. Lancet Child Adolesc Heal [Internet]. 2021 Jun;5(6):447-58. Available from: http://www.ncbi.nlm.nih.gov/pubmed/33721554

2. Jani J, Nicolaides KH, Keller RL, Benachi A, Peralta CFA, Favre R, et al. Observed to expected lung area to head circumference ratio in the prediction of survival in fetuses with isolated diaphragmatic hernia. Ultrasound Obstet Gynecol [Internet]. 2007 Jul;30(1):67-71. Available from: http://www.ncbi.nlm.nih.gov/pubmed/17587219

3. Kunisaki SM, Saito JM, Fallat ME, St Peter SD, Lal DR, Karmakar M, et al. Fetal Risk Stratification and Outcomes in Children with Prenatally Diagnosed Lung Malformations: Results from a Multi-Institutional Research Collaborative. Ann Surg [Internet]. 2020 Nov 17; Available from: http://www.ncbi.nlm.nih.gov/pubmed/33214447

4. Sandaite I, Claus F, De Keyzer F, Donè E, Van Mieghem T, Gucciardo L, et al. Examining the relationship between the lung-to-head ratio measured on ultrasound and lung volumetry by magnetic resonance in fetuses with isolated congenital diaphragmatic hernia. Fetal Diagn Ther [Internet]. 2011;29(1):80-7. Available from: http://www.ncbi.nlm.nih.gov/pubmed/20962504

5. Senat M-V, Bouchghoul H, Stirnemann J, Vaast P, Boubnova J, Begue L, et al. Prognosis of isolated congenital diaphragmatic hernia using lung-area-to-head-circumference ratio: variability across centers in a national perinatal network. Ultrasound Obstet Gynecol [Internet]. 2018;51(2):208-13. Available from: http://www.ncbi.nlm.nih.gov/pubmed/28295742

6. Jani J, Cannie M, Done E, Van Mieghem T, Van Schoubroeck D, Gucciardo L, et al. Relationship between lung area at ultrasound examination and lung volume assessment with magnetic resonance imaging in isolated congenital diaphragmatic hernia. Ultrasound Obstet Gynecol [Internet]. 2007 Nov;30(6):855-60. Available from: http://www.ncbi.nlm.nih.gov/pubmed/17932997

7. Jani JC, Cannie M, Peralta CFA, Deprest JA, Nicolaides KH, Dymarkowski S. Lung volumes in fetuses with congenital diaphragmatic hernia: comparison of 3D US and MR imaging assessments. Radiology [Internet]. 2007 Aug;244(2):575-82. Available from: http://www.ncbi.nlm.nih.gov/pubmed/17641375

8. Kim AG, Norwitz G, Karmakar M, Ladino-Torres M, Berman DR, Kreutzman J, et al. Discordant prenatal ultrasound and fetal MRI in CDH: wherein lies the truth? J Pediatr Surg [Internet]. 2019;(xxxx). Available from: https://doi.org/10.1016/j.jpedsurg.2019.11.007

9. Perrone EE, Abbasi N, Cortes MS, Umar U, Ryan G, Johnson A, et al. Prenatal assessment of congenital diaphragmatic hernia at north american fetal therapy network centers: A continued plea for standardization. Prenat Diagn [Internet]. 2021 Jan 12;41(2):200-6. Available from: https://onlinelibrary.wiley.com/doi/10.1002/pd.5859

10. Uus A, Zhang T, Jackson LH, Roberts TA, Rutherford MA, Hajnal J V, et al. Deformable Slice-toVolume Registration for Motion Correction of Fetal Body and Placenta MRI. IEEE Trans Med Imaging 
[Internet]. 2020 Feb 18;1-1. Available from: http://www.ncbi.nlm.nih.gov/pubmed/32086200

11. Cannie MM, Jani JC, Van Kerkhove F, Meerschaert J, De Keyzer F, Lewi L, et al. Fetal body volume at MR imaging to quantify total fetal lung volume: normal ranges. Radiology [Internet]. 2008 Apr;247(1):197203. Available from: http://www.ncbi.nlm.nih.gov/pubmed/18258812

12. Meyers ML, Garcia JR, Blough KL, Zhang W, Cassady CI, Mehollin-Ray AR. Fetal Lung Volumes by MRI: Normal Weekly Values From 18 Through 38 Weeks' Gestation. AJR Am J Roentgenol [Internet]. 2018;211(2):432-8. Available from: http://www.ncbi.nlm.nih.gov/pubmed/29894217

13. Petroze RT, Caminsky NG, Trebichavsky J, Bouchard S, Le-Nguyen A, Laberge J-M, et al. Prenatal prediction of survival in congenital diaphragmatic hernia: An audit of postnatal outcomes. J Pediatr Surg [Internet]. 2019 May;54(5):925-31. Available from: http://www.ncbi.nlm.nih.gov/pubmed/30786991

14. Corroenne R, Zhu KH, Johnson R, Mehollin-Ray AR, Shamshirsaz AA, Nassr AA, et al. Cost-effective fetal lung volumetry for assessment of congenital diaphragmatic hernia. Eur J Obstet Gynecol Reprod Biol [Internet]. 2021 May;260:22-8. Available from: http://www.ncbi.nlm.nih.gov/pubmed/33713885

15. Style CC, Mehollin-Ray AR, Verla MA, Olutoye OO, Lau PE, Johnson BL, et al. Accuracy of prenatal and postnatal imaging for management of congenital lung malformations. J Pediatr Surg [Internet]. 2020 May;55(5):844-7. Available from: http://www.ncbi.nlm.nih.gov/pubmed/32087934

16. King A, Lee TC, Steen E, Olutoye OO, Belfort MA, Cassady CI, et al. Prenatal Imaging to Predict Need for Urgent Perinatal Surgery in Congenital Lung Lesions. J Surg Res [Internet]. 2020;255:463-8. Available from: http://www.ncbi.nlm.nih.gov/pubmed/32622160
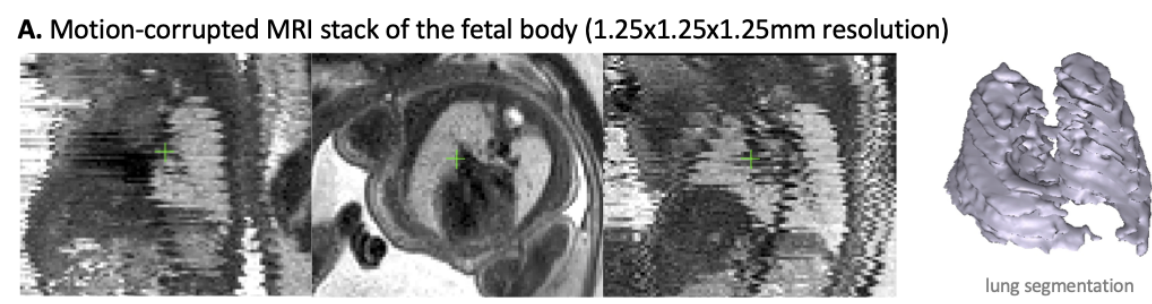

B. DSVR reconstructed 3D image $(0.85 \times 0.85 \times 0.85 \mathrm{~mm}$ resolution)
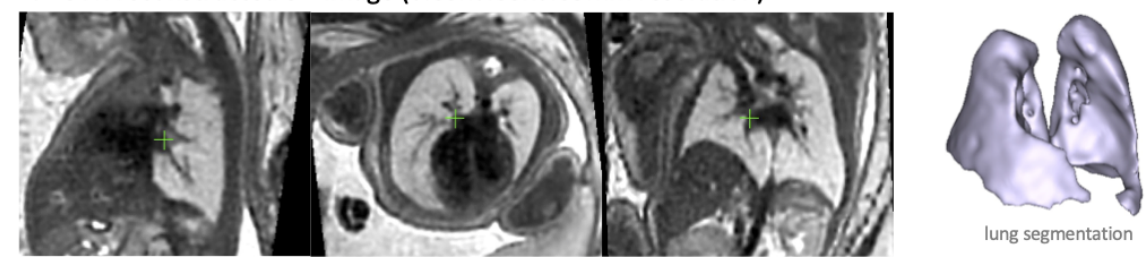
A

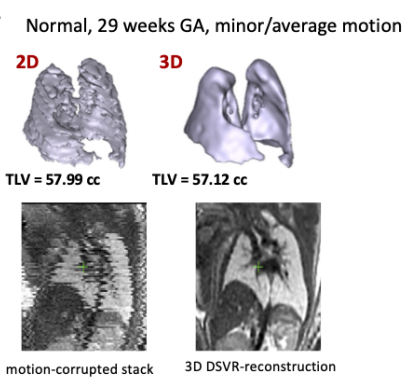

C $\mathrm{CDH}, 33$ weeks $\mathrm{GA}$, severe motion

2D
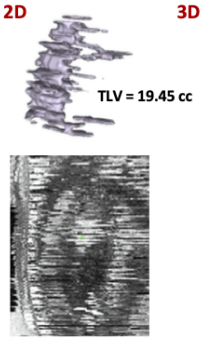

motion-corrupted stack
3D
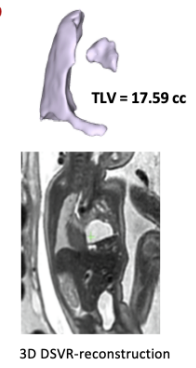

B CLL, 24 weeks GA, average motion 2D

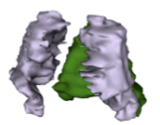

3D

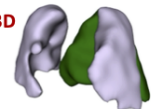

$\mathrm{TLV}=12.78 \mathrm{cc}$, lesion $=16.67$

TLV $=11.99 \mathrm{cc}$, lesion $=15.71 \mathrm{cc}$

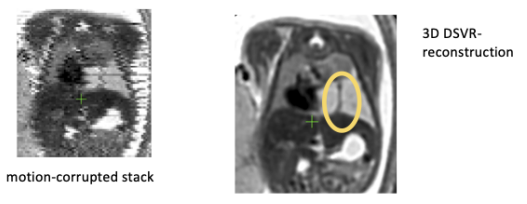

D

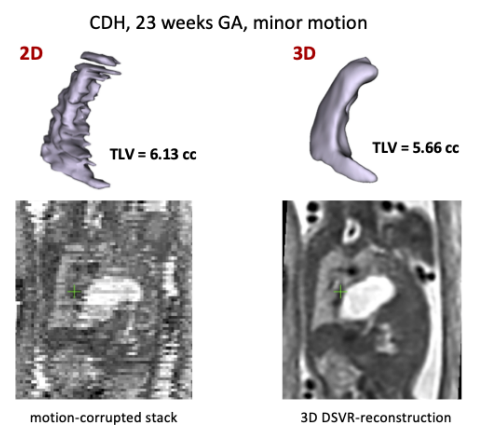

\section{D and 2D Total Lung Volumes}

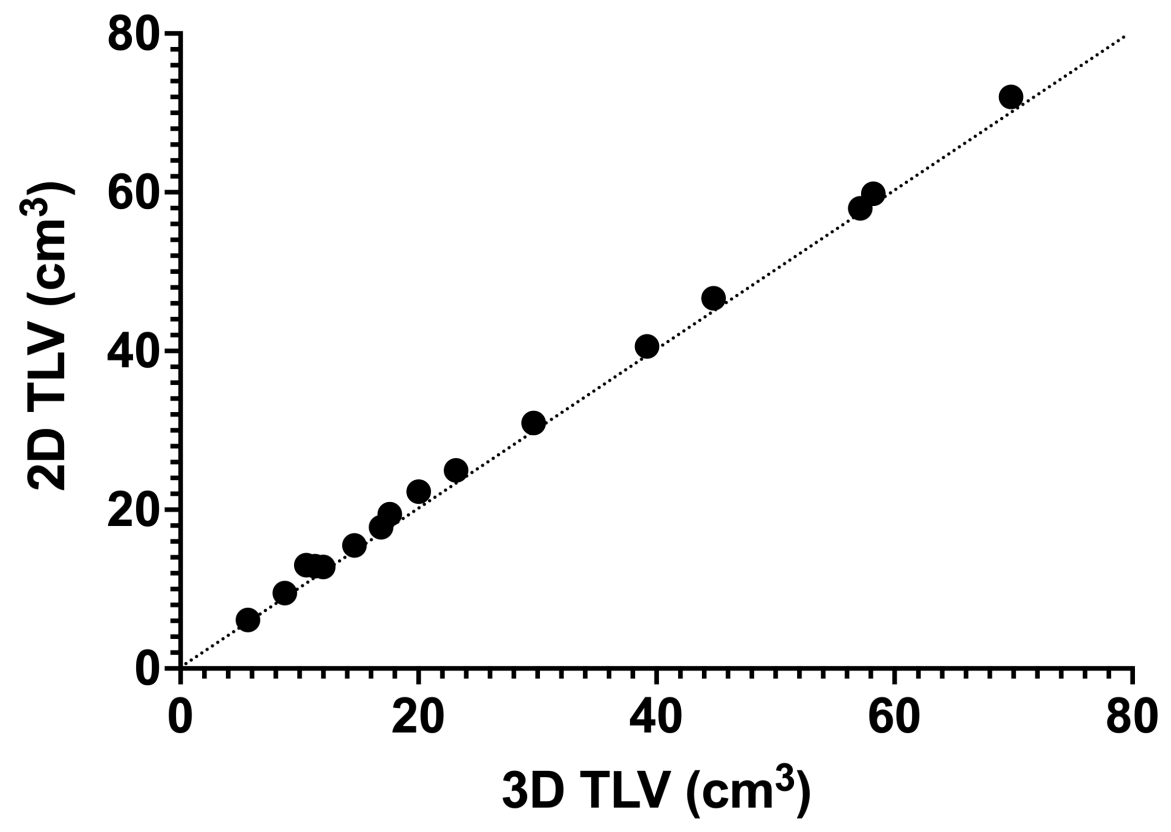




\section{Normal curve for total lung volume (TLV) [ $\left.\mathrm{cm}^{3}\right]$}
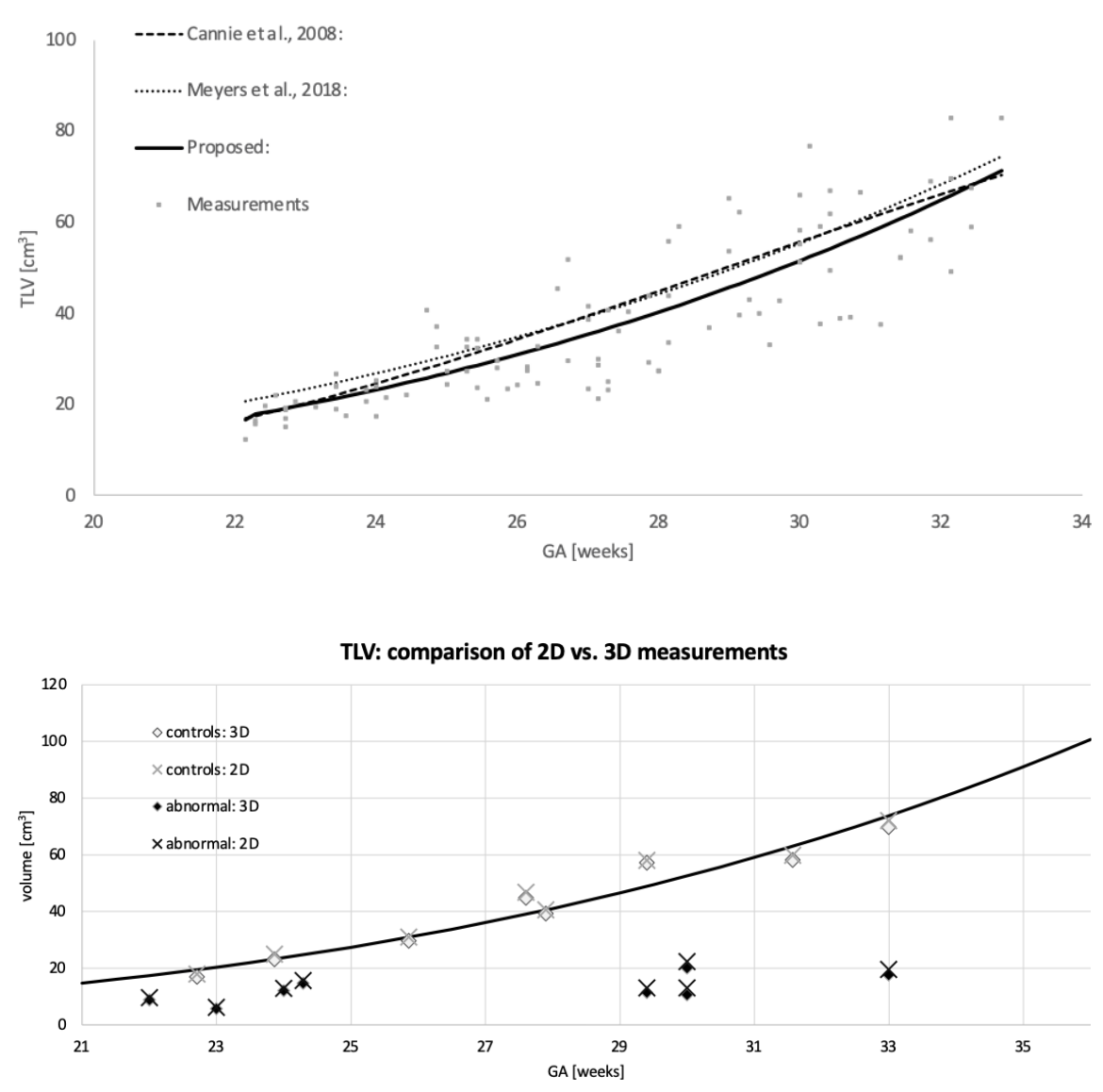

\section{Hosted file}

Table 1.docx available at https://authorea.com/users/425087/articles/538415-motion-correctedfetal-body-mri-provides-reliable-3d-lung-volumes-in-normal-and-abnormal-fetuses 